\title{
BMJ Open Prior surgical uterine evacuation of pregnancy and infertility: protocol for systematic review and meta-analysis
}

\author{
Pengcheng Tu (D) , ${ }^{1,2}$ Kaiyan Pei ${ }^{1,2}$
}

To cite: Tu P, Pei K. Prior surgical uterine evacuation of pregnancy and infertility: protocol for systematic review and meta-analysis. BMJ Open 2020;10:e034837. doi:10.1136/ bmjopen-2019-034837

\section{- Prepublication history and} additional material for this paper are available online. To view these files, please visit the journal online (http://dx.doi org/10.1136/bmjopen-2019034837).

Received 07 October 2019 Revised 18 February 2020 Accepted 04 June 2020

\section{Check for updates}

(c) Author(s) (or their employer(s)) 2020. Re-use permitted under CC BY-NC. No commercial re-use. See rights and permissions. Published by BMJ.

${ }^{1}$ National Research Institute for Family Planning, Beijing, China ${ }^{2}$ Graduate School, Chinese Academy of Medical Sciences and Peking Union Medical College, Beijing, China

Correspondence to Professor Kaiyan Pei; peikaiyan@nrifp.org.cn

\section{ABSTRACT}

Introduction Prior surgical uterine evacuation is associated with an increased risk of infertility. However, findings are inconsistent, highlighting the need for a clear consensus on the effect of prior surgical uterine evacuation on the risk of infertility. Therefore, the aim of this systematic review and meta-analysis is to summarise the available evidence examining the association between prior surgical uterine evacuation and the risk of infertility. Methods and analysis A systematic search of electronic databases (ie, PubMed, Scopus, ClinicalTrials.gov, EMBASE and ScienceDirect) will be conducted since their inception until October 2019 with no limit for language using a detailed prespecified search strategy. Both the authors will independently screen titles and abstracts and select full-text articles, perform data extraction and appraise the quality of included studies using a bias classification tool. Meta-analyses will be performed to calculate the overall pooled estimates using the generic inverse variance method. This systematic review and meta-analysis will follow the Preferred Reporting Items for Systematic Reviews and Meta Analyses (PRISMA) guidelines.

Ethics and dissemination Given that this is a protocol based on published data, there is no requirement for ethics approval. It is anticipated that the dissemination of results will be reported according to the PRISMA statement. The results will be published in peer-reviewed journals and presented at scientific conferences.

PROSPERO registration number CRD42019117266.

\section{INTRODUCTION}

In recent years, infertility remains a highly prevalent global condition. It is estimated to affect around $9 \%$ of reproductive-aged couples and as many as 186 million people worldwide with the highest infertility prevalence in South Asia, Sub-Saharan Africa, North Africa/Middle East and Central/ Eastern Europe and Central Asia. ${ }^{1-4}$ Relative contribution of various factors leading to infertility has been extensively studied but is not adequately understood. Medically, induced termination of pregnancy (I-TOP) is defined as an intervention to voluntarily terminate a pregnancy (ie, induced abortion) by either surgical or medical means, so it does not result in a live birth. ${ }^{5-8}$ Spontaneous abortion

\section{Strengths and limitations of this study}

This systematic review and meta-analysis will follow the Preferred Reporting Items for Systematic Reviews and Meta Analyses guidelines, ensuring consistency and uniformity in reporting the full systematic review.

- The review aims to provide a clear consensus on the effect of prior surgical uterine evacuation on the risk of infertility.

- Two reviewers will screen for study eligibility and perform the quality assessment to minimise the potential for reviewer bias.

- The presence of recall bias may pose a limitation for this review.

$(\mathrm{SAB})$ is defined as spontaneous intrauterine pregnancy loss prior to 20 weeks. ${ }^{6-11}$ Surgical uterine evacuation (for either I-TOP or treatment of $\mathrm{SAB}$ ) was defined as a procedure using surgical instruments, either dilation and evacuation or vacuum aspiration, to remove the fetus and placenta from the uterus. Prior surgical uterine evacuation is now recognised as an independent risk factor for preterm birth. ${ }^{611-15}$ Studies have shown that infertility is associated clinically with endometriosis, ${ }^{16}$ polycystic ovary syndrome $(\mathrm{PCOS})^{17}$ and chronic endometritis and/or adhesions that need hysteroscopic investigation. ${ }^{18}$ By comparison, the effect of prior surgical uterine evacuation on infertility has received relatively little attention.

Since induced abortion became legal in many countries around the world, there is increasing evidence suggesting that prior surgical uterine evacuation may increase the relative risk of infertility. ${ }^{19-22}$ A case-control study has also postulated that the number of uterine evacuation may influence the association with infertility. ${ }^{20}$ Conversely, other studies showed that prior surgical uterine evacuation may not be an independent risk factor for infertility, ${ }^{23-25}$ highlighting the need for a further systematic review of the 
effect of prior surgical uterine evacuation on infertility, with a view to meta-analysis of the outcomes.

Therefore, the aim of this systematic review and metaanalysis is to summarise the available evidence examining the association between prior surgical uterine evacuation and the risk of infertility. The information obtained from this review is important to urge women to realise the risk of surgical uterine evacuation and use contraceptive methods correctly and continually in order to reduce the rate of repeated abortions. It is also important to enhance our understanding of the decision support available to women regarding choices between surgical and medical abortion.

\section{Population}

The systematic review will include all studies of women with prior surgical uterine evacuation, compared with a control group without prior surgical uterine evacuation, which reported data about the subsequent fertility. Women with endometriosis, PCOS and chronic endometritis and/or adhesions who need hysteroscopic investigation will be excluded from the proposed analysis because those cases are concomitant causes of infertility.

\section{Intervention/exposures}

Study participants in the intervention group must bewomen who experienced prior surgical uterine evacuation and whose subsequent ferlitity status were reported .

\section{Comparison}

Study participants in the comparison group must be women who had never had a prior surgical uterine evacuation and whose fertility status were reported. For example, women who experienced a prior surgical uterine evacuation at least once will be compared with women who never experienced a surgical uterine evacuation. Cases managed by medical abortion will be included in the control group, but cases with expectant management will be excluded.

\section{Outcomes}

Infertility is defined as not being able to get pregnant after 1 year or longer of unprotected sex.

\section{Methods and design}

This systematic review and meta-analysis will follow the Preferred Reporting Items for Systematic Reviews and Meta Analyses (PRISMA) guidelines. ${ }^{26}$

\section{Objectives}

This study aims to conduct a systematic review and metaanalysis to examine the association between prior surgical uterine evacuation and the risk of infertility.

\section{Review question}

This systematic review will address the following research questions:

1. Does prior surgical uterine evacuation increase the risk of infertility?
2. Is there an increased risk of infertility with an increasing number of prior surgical uterine evacuation, that is, 'dose-response gradient'?

3. Is there a difference in the risk of infertility between surgical uterine evacuation and medical abortion?

\section{Criteria for considering studies for the review}

Inclusion criteria

This criteria includes case-control studies (women with diagnosed infertility and previous exposure) and cohort studies (women with uterine evacuation followed to check their fertility).

Studies will be included only if there is a comparative cohort.

We will only include information available from the publications and will not contact primary authors.

\section{Exclusion criteria}

Studies focused on women with endometriosis, PCOS and chronic endometritis and/or adhesions who need hysteroscopic investigation.

Studies without a control group.

Case reports, case series, letters, commentaries, notes, editorials and conference abstracts.

\section{Search strategy}

Electronic databases (ie, PubMed, Scopus, ClinicalTrials. gov, EMBASE and ScienceDirect) will be searched since their inception until October 2019 with no limit to language. The search terms will be modified according to database requirements. The search terms used will be the following keywords: infertility, secondary infertility, sterility, postpartum sterility, subfertility, miscarriage, uterine evacuation, abortion, induced abortion, spontaneous abortion, and termination of pregnancy, curettage, first trimester, second trimester, mifepristone, misoprostol, dilatation and evacuation, dilation and curettage. (The full search strategy is included in online supplementary file 1.)

\section{Selection of studies for inclusion in the review}

Titles and abstracts of studies retrieved from each database search will be stored and managed in EndNote reference manager. The titles and abstracts of all studies will be independently assessed by the authors (PT and KP). Full texts will be obtained where necessary to screen for eligibility in the systematic review and meta-analysis in accordance with the predefined inclusion/exclusion criteria. Discrepancies will be resolved by consensus.

\section{Risk of bias (quality) assessment}

The quality of all included studies will be independently assessed by two reviewers (PT and KP) using an established quality assessment tool for observational studies. This tool has been described in detail elsewhere. ${ }^{27}$ In summary, common features of the six types of bias most often associated with observational studies will be assessed: selection, exposure, outcome, analytic, attrition and confounding. For each study, each component will be assigned a risk 
of bias category: minimal, low, moderate, high or not reported. For example, selection bias will be minimised if the sample was taken from a 'consecutive unselected population', while conversely, a study with high selection bias will arise if sample selection is ambiguous and the sample is not likely representative. Discrepancies will be resolved by consensus.

\section{Data extraction}

Data from each eligible study will be extracted without modification of the original data onto custom-made data collection forms by two independent investigators (PT and KP) separately. Discrepancies will be resolved by consensus. Information of confounders adjusted and adjusted risk estimates will be collected when available. When necessary, we will contact authors of the studies to request for missing data, incomplete report or any uncertainties.

\section{Data synthesis and assessment for heterogeneity}

The final review will include data presented in summary tables and a narrative synthesis to present the characteristics of the included studies. The data analysis will be completed independently by both the authors (PT and KP) using meta-analytic software (Revman from the Cochrane Collaboration). ${ }^{28}$ Discrepancies will be resolved by discussion. According to the Cochrane handbook criteria, ${ }^{28}$ the Higgins $\mathrm{I}^{2}$ test will be used as a measure of heterogeneity among studies. A fixed-effects model will be used where heterogeneity is low $\left(\mathrm{I}^{2}\right.$ value of less than $50 \%$ ), and a random-effects model where heterogeneity is high ( $\mathrm{I}^{2}$ value of $50 \%$ or more) to explore the association between prior surgical uterine evacuation and infertility. Funnel plot will be used to assess publication bias if more than 10 studies are included. Egger's and Beggar's tests will also be used to check publication bias. ${ }^{29}$ Subgroup analysis will be performed on the basis of the number of prior surgical uterine evacuation, gestational weeks ( $<6$ vs $6-9$ vs $9-12$ vs $\geq 12$ gestational weeks) and the method of uterine evacuation (surgical uterine evacuation vs medical abortion). Besides, we will consider metaregression where 10 or more studies are included in our meta-analysis since it is an extension to subgroup analyses that allows the effect of continuous, as well as categorical, characteristics to be investigated and in principle allows the effects of multiple factors to be investigated simultaneously. The quality of the findings on each outcome of interest across studies will be assessed using the Grading of Recommendations, Assessment, Development and Evaluations (GRADE) guidelines, which are developed by the GRADE Working Group. ${ }^{30}$

\section{ETHICS AND DISSEMINATION}

Given that this is a protocol based on published data, there is no requirement for ethics approval. It is anticipated that the dissemination of results will be reported according to the PRISMA statement. ${ }^{31}$ The results will be published in peer-reviewed journals and presented at scientific conferences.

\section{Potential limitations}

First of all, one major concern is that infertility is a multifactorial condition that involves both individuals in a couple. However, we are unaware of any observational studies that assessed male infertility factors at the same time and whether this could influence our results.

Second, publication bias may reduce the likelihood of retrieving studies which report non-significant associations between prior surgical uterine evacuation and the risk of infertility. Search strategies for retrieving studies in electronic databases are limited, so a funnel plot will be used to assess publication bias if more than 10 studies are included.

Furthermore, the presence of recall bias is a major concern. Women tend to omit I-TOP from their medical history, which would lead to under-reporting of abortion in the control group and under-reporting of the number of abortions in the case group. And a woman electing to have an induced abortion might be content with an infertile state and not seek help for her problem. However, this woman might be more likely to go to a physician than a woman who had a successful pregnancy and then became infertile, which may contribute to overstating the risk.

Last but not least, the presence of selection bias and residual confounding is a concern in all observational studies. Potential confounders may include maternal age, marital status, social class, smoking, parity, country, ethnic group, education and family history. As mentioned above, our meta-analyses will display both crude and adjusted results where possible using the generic inverse variance method, basing the adjustment of the definition outlined in each individual study.

\section{DISCUSSION}

There is a lack of consensus on whether prior surgical uterine evacuation independently increases the risk of infertility. Although male infertility contributes to over half of all cases of childlessness globally, infertility remains a woman's social burden. ${ }^{2}$ In China, the number of induced abortions recently is reported over 9 million per year, with more than half being repeated abortions. ${ }^{32}$ This systematic review and meta-analysis will summarise the available evidence examining the association between prior surgical uterine evacuation and the risk of infertility. The results would enhance our understanding of the decision support available to women choosing between surgical and medical abortion. More importantly, we hope to urge women to realise the risk of surgical uterine evacuation and use contraceptive methods correctly and continually in order to reduce the incidence of repeated abortion.

Contributors PT and KP conceived and designed the protocol, and PT drafted the protocol manuscript. PT developed the search strategy. PT and KP planned the data extraction and quality appraisal of included studies. PT and KP critically revised the 
manuscript for methodological and intellectual content. Both the authors approved the final version.

Funding This project was supported by the CAMS Innovation Fund for Medical Sciences (CIFMS) (No 2018-I2M-1-004).

Competing interests None declared.

Patient and public involvement Patients and/or the public were not involved in the design, or conduct, or reporting, or dissemination plans of this research.

Patient consent for publication Not required.

Provenance and peer review Not commissioned; externally peer reviewed.

Open access This is an open access article distributed in accordance with the Creative Commons Attribution Non Commercial (CC BY-NC 4.0) license, which permits others to distribute, remix, adapt, build upon this work non-commercially, and license their derivative works on different terms, provided the original work is properly cited, appropriate credit is given, any changes made indicated, and the use is non-commercial. See: http://creativecommons.org/licenses/by-nc/4.0/.

\section{ORCID iD}

Pengcheng Tu http://orcid.org/0000-0001-7015-576X

\section{REFERENCES}

1 Mascarenhas MN, Flaxman SR, Boerma T, et al. National, regional, and global trends in infertility prevalence since 1990: a systematic analysis of 277 health surveys. PLoS Med 2012;9:e1001356.

2 Inhorn MC, Patrizio P. Infertility around the globe: new thinking on gender, reproductive technologies and global movements in the $21 \mathrm{st}$ century. Hum Reprod Update 2015;21:411-26.

3 Boivin J, Bunting L, Collins JA, et al. International estimates of infertility prevalence and treatment-seeking: potential need and demand for infertility medical care. Hum Reprod 2007;22:1506-12.

4 GBD 2017 Population and Fertility Collaborators. Population and fertility by age and sex for 195 countries and territories, 1950-2017: a systematic analysis for the global burden of disease study 2017. Lancet 2018;392:1995-2051.

5 ESHRE Capri Workshop Group. Induced abortion. Hum Reprod 2017;32:1160-9.

6 Saccone G, Perriera L, Berghella V. Prior uterine evacuation of pregnancy as independent risk factor for preterm birth: a systematic review and metaanalysis. Am J Obstet Gynecol 2016;214:572-91.

7 Sedgh G, Bearak J, Singh S, et al. Abortion incidence between 1990 and 2014: global, regional, and subregional levels and trends. Lancet 2016;388:258-67.

8 Kmietowicz Z. Medical abortions more common than surgery for first time in 2014 in England and Wales. BMJ 2015;350:h3177.

9 Jurkovic D, Overton C, Bender-Atik R. Diagnosis and management of first trimester miscarriage. BMJ 2013;346:f3676.

10 Spontaneous abortion. Lancet 1975;2:591-2.

11 Spong CY. Prediction and prevention of recurrent spontaneous preterm birth. Obstet Gynecol 2007;110:405-15.

12 Shah PS, Zao J. Knowledge synthesis group of determinants of preterm LBWb. induced termination of pregnancy and low birthweight and preterm birth: a systematic review and metaanalyses. BJOG 2009;116:1425-42.

13 Lemmers M, Verschoor MAC, Hooker AB, et al. Dilatation and curettage increases the risk of subsequent preterm birth: a systematic review and meta-analysis. Hum Reprod 2016;31:34-45.

14 Oliver-Williams C, Fleming M, Wood AM, et al. Previous miscarriage and the subsequent risk of preterm birth in Scotland, 1980-2008: a historical cohort study. BJOG 2015;122:1525-34.

15 Simoens C, Goffin F, Simon P, et al. Adverse obstetrical outcomes after treatment of precancerous cervical lesions: a Belgian multicentre study. BJOG 2012;119:1247-55.

16 de Ziegler D, Borghese B, Chapron C. Endometriosis and infertility: pathophysiology and management. Lancet 2010;376:730-8.

17 Reyes-Muñoz E, Sathyapalan T, Rossetti P, et al. Polycystic ovary syndrome: implication for drug metabolism on assisted reproductive techniques-a literature review. Adv Ther 2018;35:1805-15.

18 Vitale SG, Sapia F, Rapisarda AMC, et al. Hysteroscopic Morcellation of submucous Myomas: a systematic review. Biomed Res Int 2017;2017:1-6.

19 Trichopoulos D, Handanos N, Danezis J, et al. Induced abortion and secondary infertility. Br J Obstet Gynaecol 1976;83:645-50.

20 Tzonou A, Hsieh CC, Trichopoulos D, et al. Induced abortions, miscarriages, and tobacco smoking as risk factors for secondary infertility. J Epidemiol Community Health 1993;47:36-9.

21 Koster W. Linking two opposites of pregnancy loss: induced abortion and infertility in Yoruba Society, Nigeria. Soc Sci Med 2010;71:1788-95.

22 Hamy A-S, Mayenga J-M, Grefenstette I, et al. [A study comparing previous induced abortion rates in populations of newly delivered women and infertile women]. Gynecol Obstet Fertil 2008;36:395-9.

23 Lin T-B, Hsieh M-F, Hou Y-C, et al. Long-Term physical health consequences of abortion in Taiwan, 2000 to 2013: a nationwide retrospective cohort study. Medicine 2018;97:e11785.

24 Torres-Sánchez L, López-Carrillo L, Espinoza H, et al. Is induced abortion a contributing factor to tubal infertility in Mexico? Evidence from a case-control study. BJOG 2004;111:1254-60.

25 Minh PN, Vinh NQ, Tuong HM, et al. A case-control study on the relationship between induced abortion and secondary tubal infertility in Vietnam. Fukushima J Med Sci 2002;48:15-25.

26 Shamseer L, Moher D, Clarke M, et al. Preferred reporting items for systematic review and meta-analysis protocols (PRISMA-P) 2015: elaboration and explanation. BMJ 2015;350:g7647.

27 McDonald SD, Han Z, Mulla S, et al. Preterm birth and low birth weight among in vitro fertilization singletons: a systematic review and meta-analyses. Eur J Obstet Gynecol Reprod Biol 2009;146:138-48.

28 Higgins J, Thomas J, Chandler J, et al. Cochrane Handbook for systematic reviews of interventions, 2019.

29 Higgins JPT, Thompson SG. Quantifying heterogeneity in a metaanalysis. Stat Med 2002;21:1539-58.

30 Balshem H, Helfand M, Schünemann $\mathrm{HJ}$, et al. Grade guidelines: 3. rating the quality of evidence. $J$ Clin Epidemiol 2011;64:401-6.

31 Moher D, Shamseer L, Clarke M, et al. Preferred reporting items for systematic review and meta-analysis protocols (PRISMA-P) 2015 statement. Syst Rev 2015;4:1.

32 Tang L, Wu S, Li J, et al. Post-abortion family planning counselling practice among abortion service providers in China: a nationwide cross-sectional study. Eur J Contracept Reprod Health Care 2017;22:24-9. 